learning disorders. The incidence of subsequent ADHD among these infants would be of interest.

\title{
DYSMORPHIC SYNDROMES AND LEARNING DISABILITIES
}

Four cases of Kabuki make-up syndrome (KMS) from various ethnic groups in Vancouver, British Columbia, are reported from the Sunny Hill Health Centre for Children, Vancouver. Three were diagnosed late, at 7 years or older. The cardinal manifestations are: 1) dysmorphic facies - long palpebral fissures, large ears, arched eyebrows, outer one third of the lower eyelid turned outward, similar to make-up of Kabuki actors in Japanese theatre; 2) brachydactyly of 5th finger; 3) increased digital ulnar loops; 4) mental retardation; 5) short stature. All 4 patients presented early with delayed speech, all had autistic-like behavior, and 2 had repetitive stereotyped behavior. All showed variable degrees of learning disabilities and/or retardation, and 2 had declines in IQ in adolescence. (Ho HH, Eaves LC. Kabuki make-up (Niikawa-Kuroki) syndrome: cognitive abilities and autistic features. Dev Med Child Neurol July 1997;39:487-490). (Respond: Helena H Ho, MD FRCPC, Sunny Hill Health Centre for Children, 3644 Slocan St, Vancouver, BC, Canada V5M 3E8).

COMMENT. The diagnosis of Kabuki make-up syndrome should be considered in dysmorphic children with microcephaly and presenting with learning disabilities and/or autism. Although the majority of cases are Asian from Japan, KMS occurs in other Asians, Europeans, South American, Arab, Indian, and Chinese. The condition may be overlooked in Asians outside Japan, since the long palpebral fissures and short stature can be accepted as normal.

\section{OBSESSIVE-COMPULSIVE DISORDER}

\section{STRIATAL MRI ABNORMALITIES IN OCD}

MRI scans from 19 children aged 7 to 18 years, with recent onset obsessive-compulsive disorder (OCD), untreated with psychotropic drugs, and 19 matched controls, were analyzed for striatal volume at Western Psychiatric Institute, University of Pittsburgh, PA. Patients with OCD had significantly smaller putamen and total striatal volumes ( $7 \%$ reduction) and larger third ventricles than controls. Prefrontal cortical, white matter, lateral ventricular, and intracranial volumes were not altered. Reduced striatal volume was inversely correlated with severity of OCD symptoms, but not with illness duration or age of onset. Males and females were equally involved. (Rosenberg DR, Keshavan MS, O'Hearn KM et al. Frontostriatal measurement in treatmentnaive children with obsessive-compulsive disorder. Arch Gen Psychiatry Sept 1997;54:824-830). (Reprints: David R Rosenberg MD, Wayne State University School of Medicine, Psychiatry 9B, 4201 St Antoine Blvd, Detroit, MI 48201).

COMMENT. This study confirms the striatal pathology in children with obsessive-compulsive disorder, and emphasizes the role of the putamen in OCD. The findings were not related to prior medication effects or comorbid illness such as depression or anxiety.

Oculomotor response inhibition abnormalities in OCD are reported by the above authors in a study of 18 untreated children, aged 8 to 16 years, compared to 18 controls. The ability to suppress behavioral responses related to prefrontal cortical function was reduced in OCD patients. (Rosenberg DR, Averbach DH, O'Hearn KM et al. Arch Gen Psychiatry Sept 1997;54:831-838). 